University of Texas at El Paso

ScholarWorks@UTEP

\title{
Cantor's Paradise Regained: Constructive Mathematics from Brouwer to Kolmogorov to Gelfond
}

Vladik Kreinovich

The University of Texas at El Paso, vladik@utep.edu

Follow this and additional works at: https://scholarworks.utep.edu/cs_techrep

Part of the Computer Engineering Commons

Comments:

Technical Report: UTEP-CS-10-18

To appear in Proceedings of the Symposium on Constructive Mathematics in Computer Science, Lexington, Kentucky, October 25-26, 2010, Springer Lecture Notes in Computer Science.

\section{Recommended Citation}

Kreinovich, Vladik, "Cantor's Paradise Regained: Constructive Mathematics from Brouwer to Kolmogorov to Gelfond" (2010). Departmental Technical Reports (CS). 18.

https://scholarworks.utep.edu/cs_techrep/18

This Article is brought to you for free and open access by the Computer Science at ScholarWorks@UTEP. It has been accepted for inclusion in Departmental Technical Reports (CS) by an authorized administrator of ScholarWorks@UTEP. For more information, please contact Iweber@utep.edu. 


\title{
Cantor's Paradise Regained: Constructive Mathematics from Brouwer to Kolmogorov to Gelfond
}

\author{
Vladik Kreinovich \\ Department of Computer Science \\ University of Texas at El Paso \\ El Paso, TX 79968 USA \\ vladik@utep.edu
}

\begin{abstract}
Constructive mathematics, mathematics in which the existence of an object means that that we can actually construct this object, started as a heavily restricted version of mathematics, a version in which many commonly used mathematical techniques (like the Law of Excluded Middle) were forbidden to maintain constructivity. Eventually, it turned out that not only constructive mathematics is not a weakened version of the classical one - as it was originally perceived - but that, vice versa, classical mathematics can be viewed as a particular (thus, weaker) case of the constructive one. Crucial results in this direction were obtained by M. Gelfond in the 1970s. In this paper, we mention the history of these results, and show how these results affected constructive mathematics, how they led to new algorithms, and how they affected the current activity in logic programming-related research.
\end{abstract}

Keywords: constructive mathematics; logic programming; algorithms

Science and engineering: a brief reminder. One of the main objectives of science is to find out how the world operates, to be able to predict what will happen in the future. Science predicts the future positions of celestial bodies, the future location of a spaceship, etc.

From the practical viewpoint, it is important not only to passively predict what will happen, but also to decide what to do in order to achieve certain goals. Roughly speaking, decisions of this type correspond not to science but to engineering. For example, once we have come up with the design of a spaceship, once we know how exactly it will be launched, Newton's equations can predict where exactly it will be at any future moment of time - and if our goal is to reach the Moon, whether it will reach it or not. If we happen to stumble upon the design and initial conditions that satisfy our goal, great, but in practice, such situations are rare. Usually, we need to find the initial conditions (and the design) for which the spaceship will safely reach the Moon.

Similarly, once the bridge is designed, we can use the known equations of mechanics to predict its stability and vulnerability to winds. However, a more important problem is to design a bridge that will withstand the expected loads under prevailing winds. 
Resulting need for constructive mathematics. The ultimate objective of a scientific analysis is to formulate an exact mathematical model of the corresponding physical phenomena. Once the corresponding physical model is formulated in precise mathematical terms, the practical (engineering) problem can be also formulated in mathematical terms: find an object $x$ (design, trajectory, etc.) that satisfies the given precisely formulated condition $P(x)$.

At first glance, it may seem that similar statement exist in mathematics: for example, mathematicians can prove statements of the type $\exists x P(x)$. Intuitively, to claim to prove that something exists often means to actually construct the corresponding object, Indeed, for many centuries, most mathematical proofs of the existence statements were based on the actual construction. Once in a while there were proofs from contradiction, but they were rare. The situation changed drastically at the end of the 19th century. The first important result for which an existence proof was provided without an explicit construction was David Hilbert's 1888 proof of the Finite Basis Theorem [10,16-18]. This proof answered an important question (raised by a mathematician Paul Gordan from Göttingen) about the invariants of homogeneous polynomials. A polynomial $P\left(x_{1}, \ldots, x_{n}\right)$ is called homogeneous if all its monomials are of the same total degree $d$ : e.g., we can have $\sum_{i=1}^{n} a_{i} \cdot x_{i}$ or $\sum_{i, j} a_{i j} \cdot x_{i} \cdot x_{j}$, with $a_{i j}=a_{j i}$. A polynomial function $f\left(a_{\alpha}\right)$ of the coefficients $a_{\alpha}$ of this polynomial is called invariant it for every linear transformation of the unknowns $x_{i} \rightarrow x_{i}^{\prime} \stackrel{\text { def }}{=} \sum c_{i j} \cdot x_{j}$, this function changes by a multiplicative constant $f\left(a_{\alpha}^{\prime}\right)=\lambda\left(\left\{c_{i j}\right\}\right) \cdot f\left(a_{\alpha}\right)$. For example, for quadratic forms of two variables $a_{11} \cdot x_{1}^{2}+2 a_{12} \cdot x_{1} \cdot x_{2}+a_{22} \cdot x_{2}^{2}$, a discriminant $D=a_{11} \cdot a_{22}-a_{12}^{2}$ is an invariant. It was known that for the case of $n \leq 8$ variables, for every degree $d$, invariants have a finite basis in the sense that we can select finitely many invariants so that every other invariant is a polynomial of the selected ones. For example, for $n=2$ and $d=2$, every invariant is a power of the discriminant $D$. Hilbert proved that such a finite basis exists for all $n$ and all $d$, but - in contrast to the previously known proofs for $n \leq 8$ - his by-contradiction proof did not provide any actual construction of the corresponding finite set. After reading this proof, Paul Gordan himself said: "This is not mathematics; it is theology"; see, e.g., [33].

Hilbert himself provided later a constructive proof of this result $[19,20]$, but the floodgates were opened for non-constructive proofs. After Hilbert's theorem, came numerous such proofs, including proofs from Cantor's set theory. The most well-known of them was the diagonal proof that there exist irrational and transcendental numbers - the "father" of all the modern diagonalization proofs.

Indirect proofs of existence became fully accepted in the mainstream mathematics - because, strictly speaking, the existence of an object does not mean that we must be able to actually construct it. The famous topologist L. E. J. Brouwer agreed with this statement - that the existence in traditional mathematics does not imply constructibility - but he made a different conclusion: since we do need to construct objects, we must hence change the meaning of the existential quantifier in mathematics. He therefore proposed a new mathematics 
- what we now call constructive mathematics - in which the only way to prove a statement $\exists x P(x)$ is to produce a construction of an object $x$ for which $P(x)$ is true; see, e.g., $[7,8]$. Brouwer argued that this new understanding of the existential quantifiers is in better accordance with our intuition - at least with the intuition of applied mathematicians who want, e.g., to solve equations, not just "prove" that the solution exists. Because of this argument, he called his approach to mathematics intuitionism - instead of blindly following formal constructions, even when we start deviating of our intuitive meaning of the corresponding notions, we should also listen to our intuition when formal constructions lead us astray.

To illustrate Brouwer's point, it is actually not necessary to consider the case when we have infinitely many possible objects $x$. The same idea can be illustrated on the example of a simple disjunction $A \vee B$, when we have only two alternatives $A$ and $B$. For example, in classical mathematics, the Law of Excluded Middle holds, according to which every statement is either true or false $A \vee \neg A$. However, a construction interpretation of a disjunction $P \vee Q$ means that we know either $P$ or $Q$. Since we do not know the truth value of a generic statement $A$, in intuitionistic logic, the Law of Excluded Middle is not generally true.

First years of constructive mathematics: constructive mathematics as a straightjacket. Several mathematicians whose interests were close to applications agreed with (at least some of) Brouwer's ideas, the most famous of them Hermann Weyl, whose interest in application of mathematics to space-time has led him to interesting intuitionistic ideas [35].

However, such converts were rare. The main reason for this rarity is that to avoid non-constructive existence proofs, Brouwer proposed to restrict allowed logical constructions - so that proofs by contradiction become impossible. These restrictions severely limited the ability of mathematicians to prove new results. Not surprisingly, most mathematicians did not want to place themselves under such severe restrictions. The general opinion was best expressed by David Hilbert himself. By then, he has become the leading mathematician of that time. In 1900, he was tasked, by the world mathematics community, to prepare the list of most important problem that the 19 century mathematics should leave for the 20 century mathematicians to solve.

In regards to constructive mathematics, Hilbert famously said: "No one shall drive us from the paradise that Cantor has created for us."

Kolmogorov: it is the classical logic that is a straightjacket, not the constructive one. For several years, constructive mathematics and the related constructive logic were viewed as severely restricted version of the traditional ones. In logic, this view changed drastically in 1925, when the famous Russian mathematician A. N. Kolmogorov showed that classical logic can be interpreted as a subset of the constructive one. To perform such a translation, we need to interpret each classical statement $A$ as a double negation $\neg \neg A$. Correspondingly, e.g., a classical disjunction $A \vee B$ must be interpreted as $\neg \neg(\neg \neg A \vee \neg \neg B)$, etc. 
Because of Kolmogorov's result, the constructive logic was no longer perceived as a poor, limited version of the classical one: vice versa, the classical logic is a particular case of the constructive one. Thus, the constructive logic was shown to be richer and more versatile than the classical one: it allowed, e.g., in addition to the "classical" disjunction $\neg \neg(\neg \neg A \vee \neg \neg B)$, to also consider a different "constructive" disjunction $A \vee B$.

Constructive logic became a widely used, widely studied, and well-respected part of logic. But not yet constructive mathematics.

From the ubiquity of constructive logic to the ubiquity of constructive mathematics: Gelfond's groundbreaking results. In the beginning, constructive mathematics was perceived as a kind of a limited version of the classical one - the tools are limited and thus, was the argument, it is understandable that the results are limited. Even constructive mathematicians themselves originally believed in this tradeoff: yes, our theorems are not as plentiful and not as sophisticated, but they are deeper: every time we prove existence, we have a construction. Of course, the need to provide constructions limits us - but makes our results more useful in applications.

As more and more research was done in constructive mathematics, more and more results became constructively proven - and these results became more and more sophisticated. The real breakthrough came with the 1967 book of E. Bishop, a classical mathematician who "saw the light" of constructive ideas and transformed a large portion of basic math into constructive language [3].

With all these results, it became clearer that for every mathematical statement that does not explicitly contain a construction, classical truth implies constructive truth. Clearer, but still, every time, we needed to re-do the original classical proof by meticulously avoiding constructively "non-kosher" ideas (like the Law of Excluded Middle) that might have been used in the original classical proof. This re-doing was taking a lot of time and effort. A general, "meta"-result of this type was badly needed to save all this time. And this general result was proven by M. G. Gelfond in his groundbreaking papers [11-13].

Similarly to the way Kolmogorov interpreted a classical logical statement $A$ as $\neg \neg A$ and each logical connective $A \odot B$ as $\neg \neg(\neg \neg A \odot \neg \neg B)$, Gelfond interpreted classical real numbers (= classically converging sequences of integers) in the constructive terms. Specifically, a classical sequence $r_{n}$, which can be equivalently reformulated as a predicate $P(n, r)$ for which for every $n$, there is exactly one $r$ satisfying this property $(\forall n \exists ! r P(r, n)$, where ! stands for uniqueness) was constructivized into a logical property $P(n, r)$ for which $\forall n \neg \neg \exists ! r P(n, r)$ (a filling in the sense of [32]). This enabled Michael Gelfond to prove a met-result about real numbers, continuous functions, etc.

Later, with V. Lifschitz, they extended this result to a large portion of set theory - and thus working mathematics - by designing a constructive version of set theory that allowed high-level constructions similar to the classical set theories like ZF.

Because of their results, the constructive mathematics can no longer be perceived as a poor, limited version of the classical one: vice versa, the classical 
mathematics is a particular (weaker) case of the constructive one. The constructive mathematics was shown to be richer and more versatile than the classical one: it allowed, e.g., in addition to the "classical" non-constructive existential quantifier, to also consider a different "constructive" one.

Cantor's paradise was regained - and an even better constructive one was built on top of it.

How these exciting results influenced my own research. In the early 1970s, when Michael Gelfond and Vladimir Lifschitz developed their excising results in St. Petersburg, Russia, I was a student attending the logical seminars where their presented different stage of their research - and attending seminars led by them, e.g., the seminar on set theory and its possible constructivization. I learned from them (and they gave me an official A for their seminar :-), I ran my ideas by them, they helped me present my ideas and edit my papers.

My interest at that time was in using Gelfond's theorem to prove new results about constructive existence. I concentrated on three related directions.

First, since it is well known that it is not possible, in general, to compute the exact solutions to a system of equation or the exact location of a maximizing point, in practice - since measurements and implementations are approximate anyway - we only need $\varepsilon$-approximate solutions, for an appropriate accuracy $\varepsilon$. For many practical problems like solving systems of equations and finding locations of maxima, the algorithmic computability of such approximate solutions was well known. I used Gelfond's theorem to extend these results to more general problems involving integration, minimization, and maximization.

My second direction was related to the fact that the proofs of most algorithmic non-computability results essentially use functions which have several maxima and/or equations which have several solutions. It turned out that this is not an accident: uniqueness actually implies algorithmic computability. Such a result was first proven by Lacombe [31] who designed an algorithm that inputs a constructive function of one or several real variables on a bounded set that attains its maximum on this set at exactly one point - and computes this global maximum point. V. Lifschitz extended this result to constructive functions on general constructive compact spaces [32].

In my paper [25] and in my dissertation [27], I showed how this result can be applied to design many algorithms: from optimal approximation of functions to designing a convex body from its metric to constructive a shortest path in a curved space to designing a Riemannian space most tightly enclosing unit spheres in a given Finsler space [9].

The third direction was to search for other classes of mathematical statements for which classical validity automatically implies their constructive validity. I found a few such classes (e.g., functions attaining maxima at two points, with a known lower bound on the distance between them), but mostly, the results turned out to be negative. For example, Lacombe-Lifschitz-style algorithm is not possible for functions that have exactly two global maxima, not possible for functions on non-compact sets, etc. [25-29]. 
In the Appendix, we provide an informal explanation of what is so special about uniqueness, and how classes like this can be constructed.

Constructive mathematics after Gelfond: very briefly. Since the 1970s, many interesting results were constructively proved within constructive mathematics; see, e.g., $[1,2,4-6,29,30,34]$. It is impossible to enumerate all such results in this short paper. Let us just mention that the uniqueness-implies-computability results were effectively used by Kohlenbach and his school to come up with new efficient algorithms; see, e.g., [21-23].

Gelfond's research after constructive mathematics. While I was working on Michael Gelfond's constructive ideas, Michael himself moved further, in the direction that very naturally follows from his constructive research. Indeed, as we have mentioned earlier, constructive mathematics started with an observation that the meaning of disjunction $A \vee B$ (" $A$ or $B$ ") in mathematics is sometimes different from the intuitive meaning of "or". A thorough analysis of this distinction led to the realization that to adequately represent our intuition about "or", we need (at least) two different disjunction operations: the constructive disjunction and the classical disjunction.

This distinction - and constructive mathematics in general - got a great boost in the 1950s and 1960s, when computers became ubiquitous and construction became not just a theoretical concept but rather an everyday task. This boost led to the urgent need for translating the existing knowledge into algorithmic computer-understandable terms. The experience of such translation showed that "or" is not the only logical connective whose intuitive and mathematical meanings sometime differ; the same turned out to be for other connectives as well.

This realization started with the implication $A \rightarrow B$ (" $A$ implies $B$ "). In classical mathematics, $A \rightarrow B$ simply means that either $B$ is true or $A$ is false. As a result, in mathematics, statements like "if $2+2=5$ then witches can fly" make perfect sense. From the intuitive viewpoint, however, " $A$ implies $B$ " means that the statement $A$ was actually used in proving the statement $B-$ i.e., crudely speaking, that the statement $B$ would be not true without $A$. This intuitive meaning of implication was partly captured by if-then rules of logic programming.

For logic programs without negation, the intuitive meaning of commonsense implication has been captured by the notion of a minimal model, i.e., a model in which the smallest possible set of atoms is true. This means, in particular, that if $A$ is true, then either it was assumed to be true from the very beginning, or it follows from a rule of the type $A \leftarrow B, C, \ldots$ in which all the conditions $B$, $C$, etc. are already proven to be true. However, until the late $1980 \mathrm{~s}$, there was no similar intuitively clear semantics of logic program with negation. Such a semantics - called stable semantics - was provided by M. Gelfond and V. Lifschitz in their 1988 paper [14]. This semantics - in essence, formalizing the above intuitive meaning of implication - indeed proved to be very adequate in capturing 
the intuitive meaning of if-then rules, and thus, in transforming abstract commonsense and expert knowledge into a set of constructive rules - rules enabling us to algorithmically solve problems.

After the success with implication, came another realization: that the mathematical and intuitive meanings of negation are also slightly different, and that in order to capture this intuitive meaning, we need two different negations: classical negation (meaning that $A$ is known to be false) and "negation as failure" (meaning that we cannot prove $A$ ) [15].

This was just the beginning. Then came the use of disjunctions in logic programming, the use of sets, and - slowly but surely - we seem to be arriving at a situation where classical mathematics will become a particular case of this generalized "logic programming" - like it became, in effect, a particular case of constructive mathematics.

And when we reach this stage - sometime in the next century - then no one shall drive us from the paradise that Brouwer, Kolmogorov, Gelfond, Lifschitz, and others have created for us!

Acknowledgments. This work was supported in part by the National Science Foundation grants HRD-0734825 and DUE-0926721 and by Grant 1 T36 GM078000-01 from the National Institutes of Health. The author is greatly thankful to Michael Gelfond for his guidance and help, and to Tran Cao Son and Marcello Balduccini for their organization of Gelfond Festschrift.

\section{References}

1. Aberth, O.: Precise Numerical Analysis Using C++, Academic Press, New York (1998).

2. Beeson, M.J.: Foundations of Constructive Mathematics. Springer-Verlag, New York (1985).

3. Bishop, E.: Foundations of Constructive Analysis, McGraw-Hill, New York (1967)

4. Bishop E., Bridges, D.S.: Constructive Analysis, Springer, New York (1985).

5. Bridges, D.S.: Constructive Functional Analysis, Pitman, London (1979).

6. Bridges, D.S., Via, S.L.: Techniques of Constructive Analysis, Springer-Verlag, New York (2006).

7. Brouwer, L.E.J.: Over de Grondslagen der Wiskunde, Ph.D. thesis, Universiteit van Amsterdam (1907), in Dutch; English translation in Heyting, A. (ed.), Collected Works of L.E.J. Brouwer. I: Philosophy and Foundations of Mathematics, NorthHolland, Amsterdam (1975) 11-101.

8. Brouwer, L.E.J.: Über die Bedeutung des Satzes vom ausgeschlossenen Dritten in der Mathematik, insbesondere in der Funktionentheorie, Journal für die reine und angewandte Mathematik, 154: 1-7 (1924), in German; English translation: On the significance of the principle of excluded middle in mathematics, especially in function theory. In: van Heijenoort, J. (ed.), A Source Book in Mathematical Logic, 18791931, From Frege to Gödel. Harvard University Press, Cambridge, Massachusetts (1967) 334-345.

9. Busemann, H.: The Geometry of Geodesics, Dover Publ., New York (2005).

10. Cox, D.A., Little, J.B., O'Shea, D.: Ideals, Varieties, and Algorithms, SpringerVerlag, New York, 1997. 
11. Gel'fond, M.G.: On constructive pseudofunctions, Proceedings of the Leningrad Mathematical Institute of the Academy of Sciences 16, 20-27 (1969), in Russian; English translation in: Seminars in Mathematics (Published by Consultants Bureau (New York-London) 16, 7-10 (1971).

12. Gel'fond, M.G.: Relationship between the classical and constructive developments of mathematical analysis. Proceedings of the Leningrad Mathematical Institute of the Academy of Sciences 32, 5-11 (1972), in Russian; English translation in Journal of Soviet Mathematics 6(4), 347-352 (1976).

13. Gelfond, M.G.: Classes of formulas of classical analysis which are consistent with the constructive interpretation, $\mathrm{PhD}$ Dissertation, Leningrad Mathematical Institute of the Academy of Sciences (1975), in Russian.

14. Gelfond, M., Lifschitz, V.: The stable model semantics for logic programming. In: Proceedings of the Fifth International Conference on Logic Programming ICLP (1988) 1070-1080.

15. Gelfond, M., Lifschitz, V.: Classical negation in logic programs and disjunctive databases. New Generation Computing 9, 365-385 (1991).

16. Hilbert, D.: Zur Theorie der algebraischen Gebilde. Nachrichten von der Königlichen Gesellschaft der Wissenschaften und der Georg- Augusts-Universität zu Göttingen, 450-457 (1988); 25-34 and 423-430 (1889), in German.

17. Hilbert, D.: Über die Theorie der algebraischen Formen. Mathematische Annalen $36,473-534$ (1890), in German.

18. Hilbert, D.: Über die theorie der algebraischen invarianten. Nachrichten von der Königlichen Gesellschaft der Wissenschaften und der Georg- Augusts-Universität zu Göttingen 232-241 (1891); 6-16 and 439-448 (1892), in German.

19. Hilbert, D.: Über die vollen Invariantensysteme. Mathematische Annalen 42, 313370 (1893), in German.

20. Hilbert, D.: Theory of Algebraic Invariants, Cambrdige University Press, Cambridge (1993); Lecture Notes from 1897.

21. Kohlenbach, U.: Theorie der majorisierbaren und stetigen Funktionale und ihre Anwendung bei der Extraktion von Schranken aus inkonstruktiven Beweisen: Effektive Eindeutigkeitsmodule bei besten Approximationen aus ineffektiven Eindeutigkeitsbeweisen, Ph.D. Dissertation, Frankfurt am Main (1990), in German.

22. Kohlenbach, U.: Effective moduli from ineffective uniqueness proofs. An unwinding of de La Vallée Poussin's proof for Chebycheff approximation. Annals for Pure and Applied Logic 64(1), 27-94 (1993).

23. Kohlenbach, U.: Applied Proof Theory: Proof Interpretations and their Use in Mathematics. Springer Verlag, Berlin-Heidelberg (2008).

24. Kolmogorov, A. N.: O printsipe tertium non datur. Matematicheskij Sbornik 32: 646-667 (1925), in Russian; English translation: On the principle of excluded middle. In: A Source Book in Mathematical Logic, 1879-1931, van Heijenoort, J. (ed.), From Frege to Gödel. Harvard University Press, Cambridge, Massachusetts (1967) 414437.

25. Kreinovich, V.: Uniqueness implies algorithmic computability, Proceedings of the 4th Student Mathematical Conference, Leningrad University, Leningrad, 19-21 (1975), in Russian.

26. Kreinovich, V.: Reviewer's remarks in a review of Bridges, D.S.: Constrictive functional analysis, Pitman, London (1979); Zentralblatt für Mathematik 401, 22-24 (1979).

27. Kreinovich, V.: Categories of space-time models, Ph.D. dissertation, Novosibirsk, Soviet Academy of Sciences, Siberian Branch, Institute of Mathematics (1979), in Russian. 
28. Kreinovich, V.: Physics-motivated ideas for extracting efficient bounds (and algorithms) from classical proofs: beyond local compactness, beyond uniqueness.In: Abstracts of the Conference on the Methods of Proof Theory in Mathematics, MaxPlanck Institut für Mathematik, Bonn, Germany, June 3-10 (2007) p. 8.

29. Kreinovich, V., Lakeyev, A., Rohn, J., Kahl, P.: Computational complexity and feasibility of data processing and interval computations, Kluwer, Dordrecht (1998).

30. Kushner, B.A.: Lectures on Constructive Mathematical Analysis, Amer. Math. Soc., Providence, Rhode Island (1984).

31. Lacombe, D.: Les ensembles récursivement ouvert ou fermés, et leurs applications à l'analyse récurslve. Compt Rend. 245(13), 1040-1043 (1957).

32. Lifschitz, V.A.: Investigation of constructive functions by the method of fillings, J. Soviet Math. 1, 41-47 (1973).

33. Noether, M.: Paul Gordan, Mathematisce Annalen 75, 1-41 (1914).

34. Pour-El, M., Richards, J.: Computability in Analysis and Physics. Springer-Verlag, New York, (1989).

35. Weyl, H.: Das Kontinuum. Veyt, Leipzig (1918), in German; English translation: The Continuum: A Critical Examination of the Foundation of Analysis, Dover Publ., New York (1994).

Appendix: Explanation of why uniqueness naturally appears and how other possible classes of statements can be thus generated. Let us explain why uniqueness naturally appears in our attempts to describe classes of properties $P(x)$ for which the classical validity of the existence statement $\exists x P(x)$ implies its constructive validity.

To find such classes, let us try to describe all possible reasonable classes of properties $P(x)$. It is, in general, algorithmically impossible to construct an object $x$ that satisfies the given property $P(x)$; thus, instead, we may look for an object that satisfies the given only property only "approximately" (in some reasonable sense). To formally describe the notion of an approximation, we also need relations like $d(x, y) \leq \varepsilon$, meaning that the two points $x$ and $y$ are $\varepsilon$-close. To describe a general class, we can combine the atomic properties $P(x)$ and $d(x, y) \leq \varepsilon$ by using propositional connectives and quantifiers.

We need at least two different variables $x$ and $y$ to meaningfully use the formula $d(x, y) \leq \varepsilon$. For simplicity, let us restrict ourselves to the case when there are no quantifiers (other than implicitly assumed universal quantifiers in front of the formula) and that we have exactly two different variables in a formula describing the class.

We are looking for classes of classical (non-constructive) formulas, so the propositional connectives should also be understood in terms of the classical logic. In the classical logic, every propositional statement can be described in a CNF form, as a conjunction $C_{1} \& C_{2} \& \ldots$ of clauses $C_{i}$, and every clause $C_{i}$ is a disjunction of literals (i.e., atomic statements or their negations). Thus, every possible class of properties described by such formulas is an intersection of classes corresponding to clauses. So, to study general classes, it is sufficient to study classes described by individual clauses.

In the classical logic, every clause $a \vee b \vee \ldots \vee c$ can be equivalently described as a rule $\neg a \& \neg b \& \ldots \rightarrow c$. So, instead of studying clauses, we will study possible rules. 
Rules must relate to the original property, thus, one of the literals in the clause must be $P(x)$ or $\neg P(x)$. Since we must have an approximation - otherwise, no general algorithm is possible - at least one other literal must come from the atomic statement $d(x, y) \leq \varepsilon$. For this literal to be meaningful, we must have at least one literal with the variable $x$ and at least one with the variable $y$. These clauses can only contain literals coming from the atoms $P(x), P(y)$, and $d(x, y) \leq \varepsilon$. Let us classify the corresponding rules.

One of these rules to uniqueness: the rule $P(x) \& P(y) \rightarrow d(x, y) \leq \varepsilon$. Indeed, this rule means that all the solution to our problem are $\varepsilon$-close to each other i.e., that, in effect, with the accuracy $\varepsilon$, we have a unique solution. Let us show that other possible rules do not lead to meaningful classes.

If one of the literals corresponding to $P(x)$ is positive, we can make it a conclusion of the corresponding if-then rule. Depending on whether each of the remaining two literals is positive or negative, we have four possibilities:

- The first possibility is $P(x) \& d(x, y) \leq \varepsilon \rightarrow P(y)$; in this case, once $P(x)$ holds for some object $x$, it holds for all $\varepsilon$-close values $y$. In the usual case when the set of objects is a connected set (e.g., the set of real numbers, $R^{n}$, the class of all continuous or differentiable functions), in which we can get from each point $x$ to every other point $y$ by a sequence of $\varepsilon$-neighbors, this means that if $P(x)$ holds for one object $x$, it holds for every $x$ as well. Thus, this case covers only two trivial properties $P(x)$ : the property that is always true and the property that is always false.

- The second possibility is the rule $\neg P(x) \& d(x, y) \leq \varepsilon \rightarrow P(y)$. In this case, for $y=x$, we conclude that $\neg P(x) \rightarrow P(x)$ thus, that $\neg P(x)$ is impossible, and $P(x)$ holds for all $x$ - also a trivial case.

- The third possibility is $P(x) \& d(x, y)>\varepsilon \rightarrow P(y)$. Similarly to the first possibility, we can also usually connect every two elements by a sequence in which every next one is $\varepsilon$-far from the previous one, so we also only get two trivial cases.

- The fourth possibility is $\neg P(x) \& d(x, y)>\varepsilon \rightarrow P(y)$, i.e., equivalently, $\neg P(x) \& \neg P(y) \rightarrow d(x, y) \leq \varepsilon$. In this case, all the objects that do not satisfy the property $P(x)$ are $\varepsilon$-close. So, with the exception of this small vicinity, every object satisfies the property $P(x)$. In this sense, this case is "almost" trivial.

Finally, let us consider the clauses in which literals corresponding to both atoms $P(x)$ and $P(y)$ are negative. In this case, we have two possibilities:

- the possibility $P(x) \& P(y) \rightarrow d(x, y) \leq \varepsilon$ that we already considered, and

- the possibility $P(x) \& P(y) \rightarrow d(x, y)>\varepsilon$; in this case, taking $x=y$, we conclude that $P(x)$ is impossible, so the property $P(x)$ is always false.

For the case of two variables $x$ and $y$, the (informal) statement is proven. For a larger number of variables, we can have clauses of the type

$$
P(x) \& P(y) \& P(z) \rightarrow(d(x, y) \leq \varepsilon \vee d(y, z) \leq \varepsilon \vee d(x, z) \leq \varepsilon)
$$

corresponding to the assumption that there are exactly two objects satisfying the property $P(x)$, etc. 